\title{
Applications of Virtual Reality in the manufacturing industry: from design review to ergonomic studies
}

\author{
Guillaume Moreau ${ }^{1, a}$, Philippe Fuchs ${ }^{2}$ and Panagiotis Stergiopoulos ${ }^{2}$ \\ 1 CNRS, CERMA, UMR 1563, EAN - rue Massenet, BP 81931, 44319 Nantes Cedex 3, France \\ 2 Robotics Center, École des mines de Paris, 60 boulevard Saint-Michel, 75272 Paris Cedex 06, France
}

Received 30 June 2003, Accepted 20 October 2003

\begin{abstract}
Virtual reality shows to be of use in several domains of manufacturing as a complementary tool to CAD/CAM environment. Design review is one of the very first application of VR in product design, but assembly/disassembly studies are also existing though they require some more specific work. But it can also be useful beyond the scope of common CAD software such as ergonomic studies. We will show an example from the RNTL PERF-RV project where we study the ergonomics of a car dashboard and more specifically of a push-button. We will discuss the issues raised by studying ergonomics thanks to Virtual Reality and force-feedback systems which are both hardware and software.
\end{abstract}

Key words: Virtual reality / force-feedback / CAD / design review / ergonomics

Résumé - Applications de la réalité virtuelle dans l'industrie manufacturière : de la revue de projet aux études d'ergonomie. La réalité virtuelle a montré son utilité dans plusieurs domaines de l'industrie manufacturière en tant que complément aux environnements de CAO/FAO. La revue de projet est la toute première application de réalité virtuelle en conception de produit. Il existe aussi des applications de montage/démontage même si celles-ci requièrent des travaux plus spécifiques. De nouvelles applications émergent au-delà de la sphère traditionnelle des logiciels de CAO comme dans le domaine des études d'ergonomie. Nous montrerons un exemple du projet PERF-RV du RNTL où nous étudions l'ergonomie d'une planche de bord automobile et en particulier celle d'un bouton poussoir. Nous discuterons les questions soulevées par les études d'ergonomie utilisant les techniques de réalité virtuelle et de retour d'effort qui relèvent à la fois du matériel et du logiciel.

Mots clés : Réalité virtuelle / retour d'effort / CAO / revue de projet / ergonomie

\section{Introduction}

The term Virtual Reality (VR) has appeared more than twenty years ago now, but it has been far less years that VR has begun to be used efficiently in the manufacturing industry, and especially in the design phase. The magnificent claimed productivity gains have generally not been reached but VR has shown to be of use in many domains and allows to reach a better productivity though at the cost of heavy hardware, software and cultural investments. Yet, a lot of domains remain to be explored and some major issues whether in the software, hardware or cultural domain are far from being addressed.

In this paper we will overview the several applications of VR in product design and manufacturing thanks to a couple of applications. Afterwards, we will show how VR

\footnotetext{
a Corresponding author:

guillaume.moreau@ec-nantes.fr
}

could be helpful in other applications linked to product manufacturing beyond the design phase and try to discuss the technological challenges induced by these new applications as well as the possible overcoming of the limits of present applications. We will describe emerging applications of VR such as the study of ergonomics by showing that VR might overcome some current limitations of traditional ergonomic software. We will particularly tackle the subject of the use force-feedback (FF) in ergonomic applications. This is the theme of our participation to the PERF-RV project of the RNTL framework and we will lastly present our early works and results on behalf of this project.

\section{VR in design and manufacturing}

The most common use of virtual reality in the design phase is for styling review. Many examples can be found 


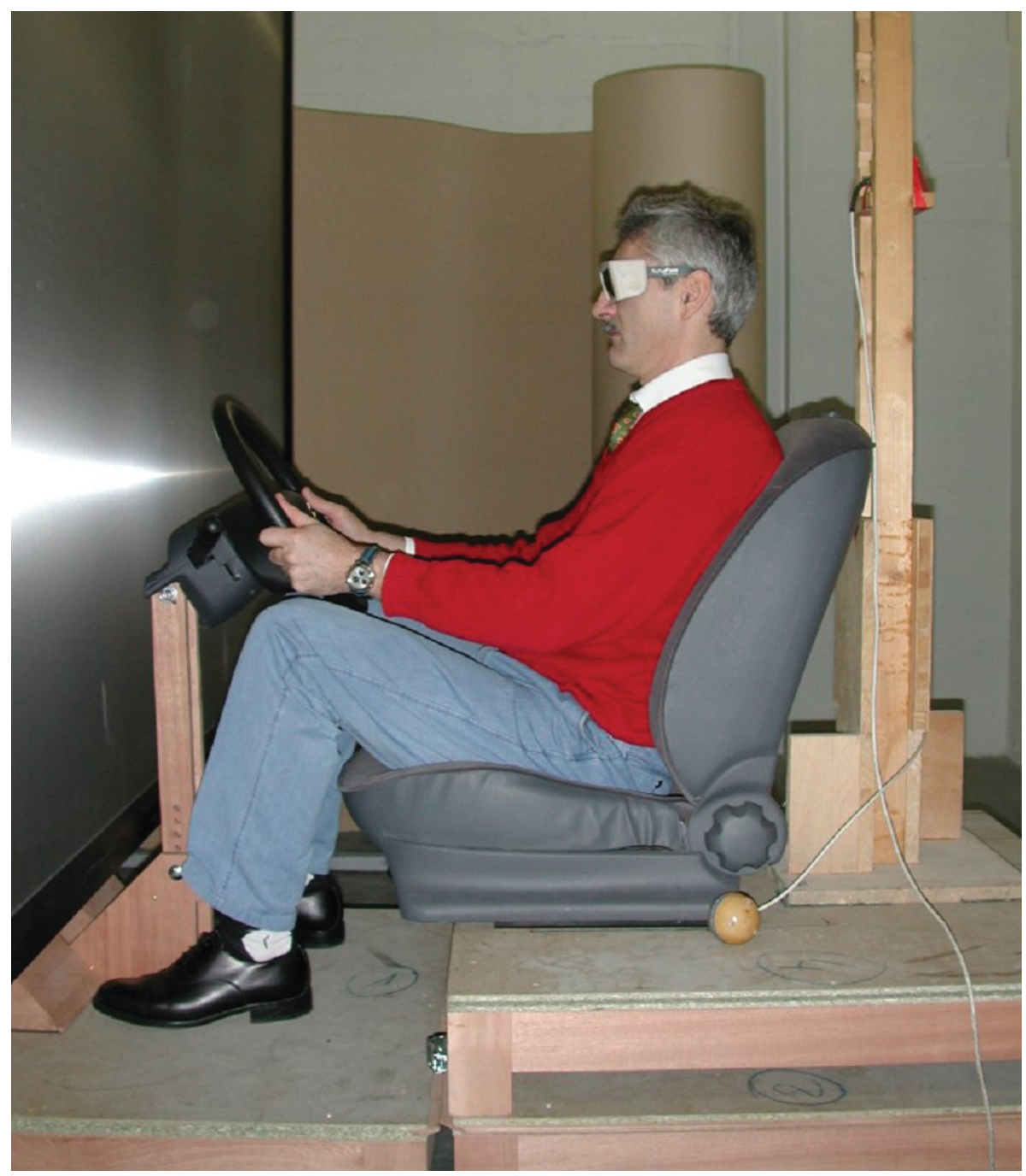

Fig. 1. The IMAVE system.

in the literature [1]. Design review basically consists in gathering several people holding different responsibilities around a mockup of a future product and taking decisions. What is interesting is the type of the decisions that are taken during these meetings.

\subsection{Non-numeric evaluation: design review}

References [1] and [2] show the example of a case study that we have performed with a car dashboard manufacturer. The key issue was to identify the functions of a design review in their particular business. The following functions were identified: visualization of different design solutions, visualization of gaps between surfaces, check of the non-visibility of technical parts (mounting screws should not be visible from driver and passenger position), and accessibility checking. The matter of this example is to show that all of the functions of design review are related to non-numeric evaluation of the prototype, and more generally to subjective evaluation. A photo of the IMAVE design review system that we have designed for
Sommer Allibert Automotive Industry is shown in Figure $1 . \mathrm{VR}$ is here the complementary approach to CAD and simulation, which perform the numeric evaluation. A virtual representation of the prototype is used instead of a physical prototype. This virtual prototype is directly derived from the Digital Mock-up (DMU) used for CAD though it is generally not an easy problem [3], as we will see later in this article. Despite this drawback, VR models are easier and cheaper to build than physical prototypes and might be easily modified thanks to the decision taken during design reviews.

Let us note that the main aim of design review is not (at this stage at least) to replace physical prototypes but to limit their number by detecting as early as possible major design flaws.

\subsection{Assembly and disassembly tasks}

Once the product has been designed, it has to be produced and maintained... The requirements are now a 
bit different. VR might be used to teach how to assemble and disassemble the product. People of the PERFRV project [4] in France have a large activity on the subject. An action leaded by Aerospatiale Matra CCR (SP3/A4) in collaboration with INRIA, Dassault Aviation and CEA consists in transposing the assembly of parts from the physical mockup to virtual reality, the use of a classical CAD workstation being completely impossible. CAD/CAM environments only allow to perform the physical accessibility of a part, i.e. can the screwdriver go through the pipes to add a little new part? They can't explain neither how to do it nor whether it is easy to do or not. The latter belongs to another domain where VR might be useful: ergonomics.

Though already at use in major car manufacturing companies like Opel, the VR technology for assembly is still the object of recent research [5] for it is the cause of major research problems (see [6] for an introduction).

\subsection{Hardware and software issues}

In the examples we have seen so far, the requirements can be summed up as visualizing a new product (and/or its part) at a 1:1 scale with the required precision and being able to interact with fixed 3D models (in most cases, simply manipulating objects in 6 dof space). Yet, these tasks seem fairly simple to describe but are already the source of numerous problems.

\subsubsection{Visualization}

The human eye is such an accurate tool that it is particularly difficult to reach its acuity: $1^{\prime}$ of a degree. This means that in order to be as accurate as the human eye on the width of a car dashboard seen from driver position, the system would require as many as 9000 pixels on a $1.5 \mathrm{~m}$ wide-screen [2]! Today's' graphics cards, projectors and obviously HMDs are far from there.

Depth perception is another important issue: though not required (see [7], pp. 108-114) stereoscopic imaging can enhance depth perception as long as it is correctly used which is generally not the case [2]. There are a few constraints to fulfill in order to setup correct stereoscopic vision, that does not provide the user with a strong headache:

- Relation between accommodation and convergence: stereoscopic vision was first explained in 1838 by Wheatstone [8]: the brain receives two different images that are fused into one representation. The brain retroactively acts on the eyes: first by commanding the muscles that rotate the eyes for convergence, and second by commanding muscles that deform the curvature of the lens for accommodation (the distance at which we look). On computer screens, the notion of convergence is conserved (relative orientation of the cameras) but the accommodation is fixed as the distance between the eye and the screen. Thus there is a breakdown between convergence and accommodation. In order to fuse the stereoscopic images, experience shows that it is better to use the lowest values of parallax (angular difference between two homologous points in both images) compatible with a good depth perception. In [9], it is shown that it should be limited to 1.5 degrees.

- Parallel or convergent cameras? Intuitively, we would set the cameras to converge, just as the human eyes do. Unfortunately, not only it would require eye-tracking to be really efficient (the eyes move very fast and so does the convergence angle between the eyes), but this also creates some vertical parallax, i.e. vertical shifts between homologous points in the two images. Vertical parallax is far more difficult to bear for the brain than horizontal one and causes severe eyestrain.

We come to the following main constraints for a good stereo visualization system:

1. The main object should have a low parallax (ideally null).

2. Horizontal parallax should be limited to $1.5^{\circ}$.

3. Vertical parallax should be avoided (implies parallel cameras).

\subsubsection{From CAD model to VR model}

CAD software as well as computer graphics software mainly work on non-polygonal surfaces. Unfortunately, due to real-time constraints VR software and 3D graphics cards render polygonal meshes. A conversion step (called tessellation) is then required. The tessellation problem can be basically divided in two sub problems: the first is the tessellation of a single surface (which is generally not too complicated when it has to be manufactured afterwards) and the tessellation of several surfaces that are constrained. The most classical example of tessellation problem is tessellation of two adjacent surfaces. These surfaces are separately tessellated into two different polygonal meshes, but the borders are not common any more for the tessellation algorithms have no reason to choose the same control points (see in Fig. 2).

This specific problem is easily solved by adding constraints on the tessellation or by moving the control points of the tessellated borders. This raises the more general problem of a quality of a given tessellation, i.e. the choice of a quantity $\varepsilon$ for which a model $\mathbf{M}$ gives a minimal triangulation $\mathbf{T}$ such as $\|\mathbf{T}-\mathbf{M}\| \leq \varepsilon$. On the one hand, the ideal algorithm does not exist, and on the other hand, the visual qualification of a triangulation ( $\varepsilon$ and the norm $\|$.$\| )$ is a difficult problem. In a more general way, a tessellation is obviously a compromise between its accuracy, the number of polygons generated and the time (CPU time or operator time) spent on it.

\subsubsection{Manipulation and navigation}

At this stage the virtual representation of the world is mainly visual. Special devices like electromagnetic 


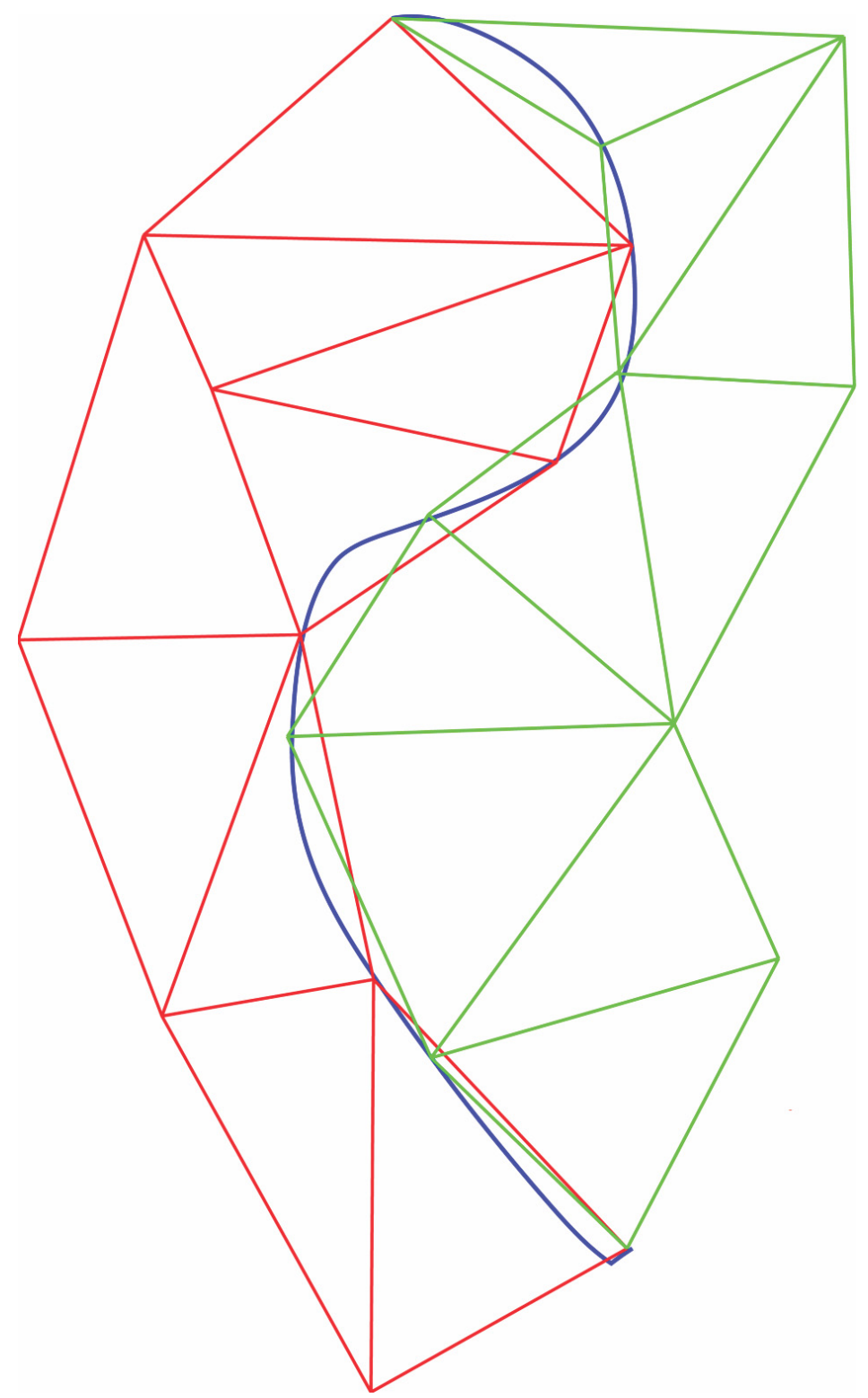

Fig. 2. Problems appearing during the tessellation of two adjacent surfaces.

trackers may be used to navigate though the virtual objects or to manipulate parts of products. Basically navigation and manipulation techniques may be divided in two categories:

- Metaphoric devices such as 2D or 3D mouse. Though they require less investment, a learning phase of the interface is mandatory and by immediate consequence the motions of the human operator cannot be the same as in real conditions.

- Natural devices such as 6D trackers (electromagnetic for instance) provide the operator with some more natural motions and of course no need to learn how to use the interface.

However, in some applications, the lack of haptic and tactile feedback will prevent the operator from learning his real task. In the next paragraphs, we will present our works on virtual ergonomics studies and the need of the introduction of force-feedback. We will discuss the hardware and software issues of force feedback for ergonomic studies.

\section{Virtual ergonomics}

\subsection{Context}

We can make a parallel between design review and ergonomic studies. If performed with physical mockups, the latter take place too late in the design process and modifications are impossible without heavy costs. The idea is then to use the digital mockup instead of the physical one thanks to ergonomics software (see for instance in Fig. 3). These software are able to sit a virtual mannequin 


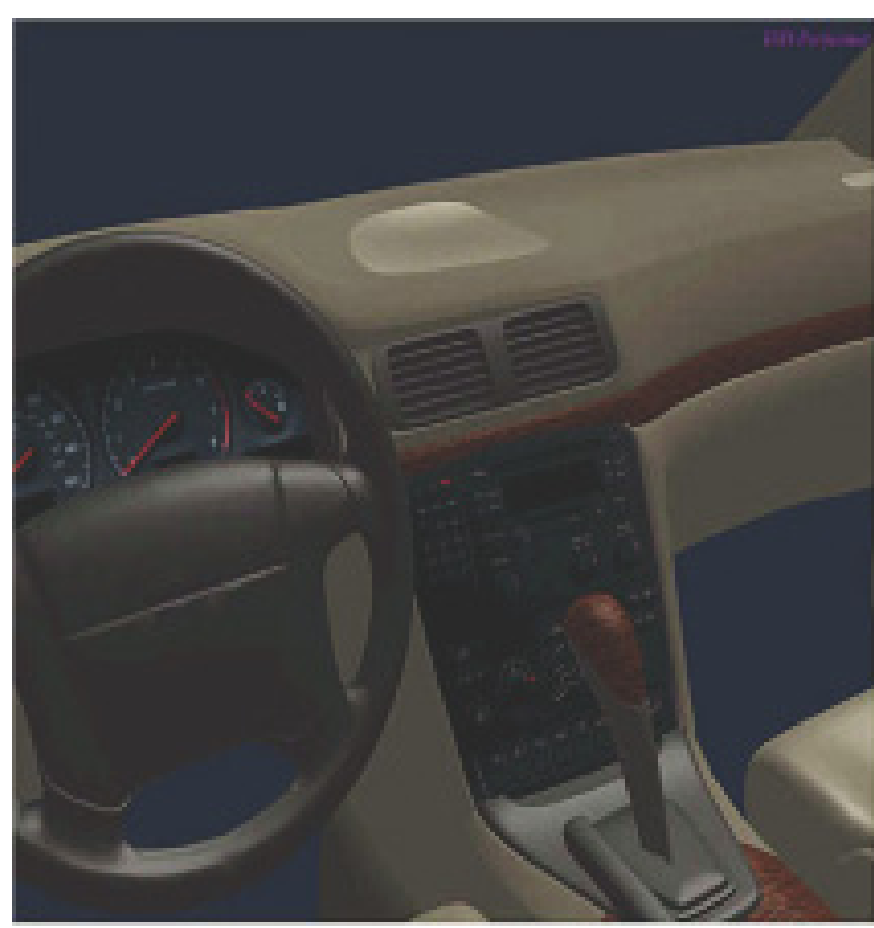

Fig. 3. Virtual ergonomics studies (Volvo).

(Fig. 4) representing a given type of population (based on anthropometrical databases) into the driver position and to perform the moves required to reach all the commands of the car. The software computes the angles of the articulations and assesses whether the command is reachable and comfortable to use.

\subsection{Current limitations of ergonomic software}

Ergonomic software represent a great step forward though they inherently translate comfort and feelings into numerical values that are only understandable by highly trained specialists. On the technical side, their main limitations are the following:

- Collision with non fixed objects or multiple collision points (for instance, one often touches the dashboard to put one's finger in more stable position to manipulate the small pushbuttons of the car radio) is not possible: it is not possible to touch or push something to reach anything else.

- Dynamics are generally not taken into account. Postural analysis is purely static and in the best case motions are interpolated thanks to inverse kinematics or energy minimization algorithms that might not be faithful to human activity. Moreover, simple equilibrium conservation is simply an ongoing project in Ramsis.

- After several meetings with the ergonomists, it appeared quite clearly that ergonomic validation was mainly performed in driving situation...

- Learning and appropriation of ergonomic software is a very complex task, estimated to 2 years [10].

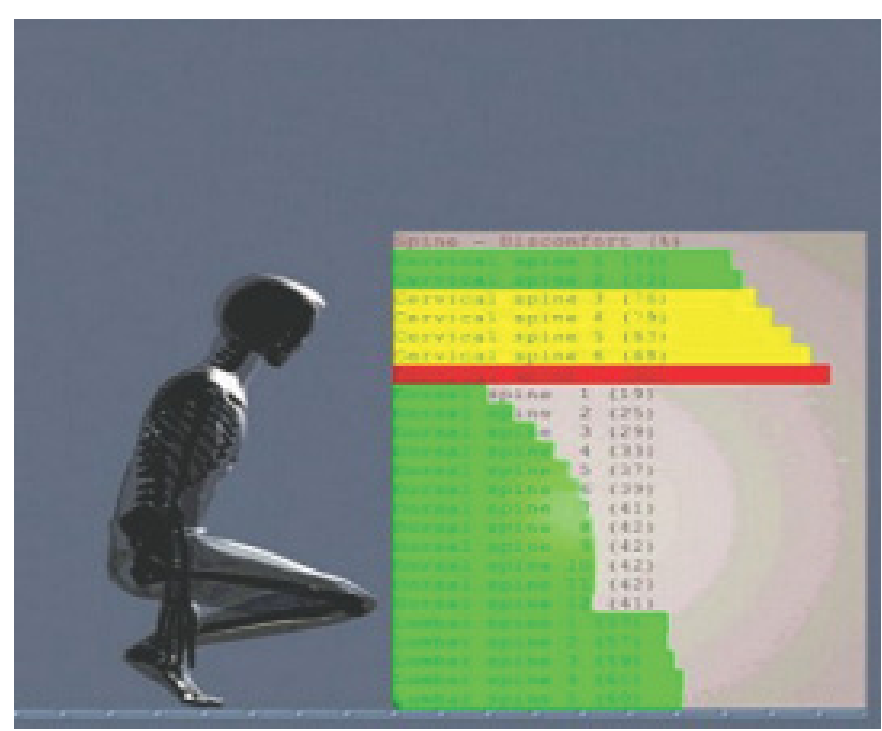

Fig. 4. Virtual mannequin (Anthropos ErgoMax).

The main drawback of classical ergonomic software such as Safework or Jack is by nature their lack of haptic feeling. The tester is not able to validate the sensation of comfort associated to the commands of the dashboard. This is also true for the IMAVE system which we have designed for design review and basic ergonomic studies. In this context, a large project was started in 2001 with a French car manufacturer and the CEA/LIST (specialized in force feedback systems) to address the following issue: to what extent can the force-feedback systems be of use to study the ergonomics of a car? In the next paragraphs, this study is briefly presented.

\subsection{Force-feedback for ergonomic studies}

\subsubsection{Requirements}

The requirements for the ergonomic studies of a car dashboard at the haptic level are pretty complex. We want to be able to test:

- The subjective feeling associated to a command out of its context of use.

- The facility of use for the driver from his normal driving position.

- The facility of use during driving.

It is then fairly important to:

- Reproduce the driving environment with a 1:1 scale!

- Choose a system powerful enough to compute haptic and visual feedback at the required rate.

- Choose the FF interface that would allow all required manipulations and able to apply a force on any part of the body involved.

\subsubsection{Considerations about force-feedback devices}

At this time, this ideal FF interface does not exist, there are technological and economical limitations that 


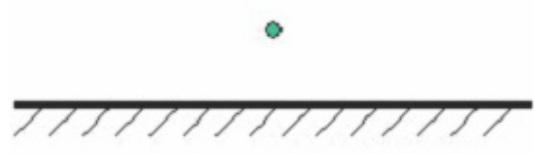

(a)

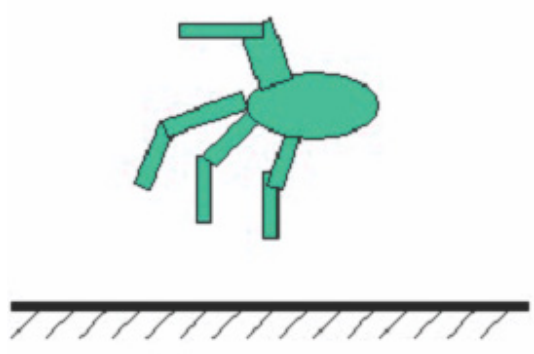

(c)

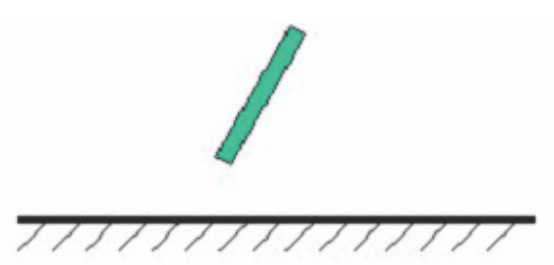

(b)

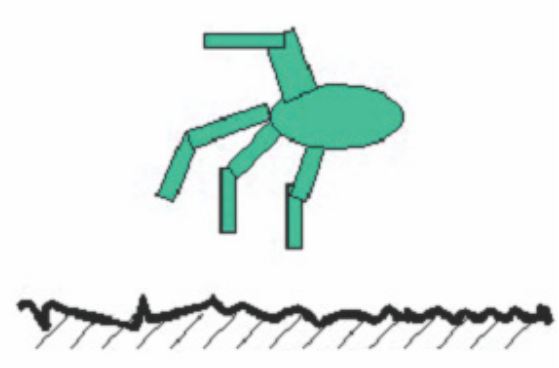

(d)

Fig. 5. Levels of contact in haptic simulation.

must be taken into account. As shown in Figure 5, there are four levels of haptic feedback:

- Punctual contact: it is the most simple case of haptic feedback using "low-cost" devices such as the Phantom Desktop from Sensable Technologies. It only allows to touch a surface and to assess the accessibility of the virtual environment.

- Contact with a solid object: in this case, the device allows the simulation of the use of a tool by simulating forces and torques, but unfortunately not of the entire hands (at most a single finger).

- FF for the naked hand: systems like the Cyberforce of Immersion Corp. allow FF on each finger and are able to simulate hand manipulation of objects, though they are very limited in terms of forces types and values.

- Tactile feedback: with tactile devices it is possible to feel some characteristics such as texture, temperature... It can be interesting to try to use them in combination with a more classical FF system in order to completely reproduce the feeling of touch.

Our first experiment is decomposed into three different steps: at first we will try to simulate the force-feedback of a simple push-button, that we will then put into its right position and at least perform the experiment during a driving task (this step requires an implementation on a driving simulator).

\subsubsection{Human factors}

It is essential to take into account the human user while designing the Virtual Environment (VE). The level of his perception outlines the level of realism required.
In the case of use of force feedback, a correct model of the haptic loop should contain a dynamic model of the user, as his existence and actions affect decisively the total performance of the system.

There have been several studies of the JND (Just Noticeable Difference) of the intensity of certain stimuli applied (or caused) and sensed by a human [11-13]. We have pursued the same goal, that is the definition of JND when comparing the force and translation between a real and a controllable button. For achieving the first experiment (see below) we asked the tester to compare a button of constant nominal translation and force, with a button whose characteristics vary each time.

\subsubsection{Collision detection}

The main objective of virtual ergonomic studies is to enable a user to sit in driver position into a virtual environment including a dashboard and its commands (just as in design review) and to let him assess the reachability and ease of use of these commands. To do this, we need to put all elements in place in the virtual environment and to be able to detect contact between the user and every part of the dashboard and its components. It both collision detection algorithms pretty efficient and specific devices to render the detected collisions. It is generally admitted that the haptic loop should run at a frequency equal or superior to $1 \mathrm{kHz}$ for simulating contact with solid objects [14]. We have developed some specific algorithms for fast collision detection [15] between a point and a virtual environment and are currently working on collision detection between different basic primitives and the VE based on the ERIT framework developed by Martin 
Held at the University of Salzburg [16]. Results should be published very soon [17].

In the IMAVE system, we have used dashboard models of about 400000 polygons. Our first algorithm can sustain a $2.8 \mathrm{kHz}$ rate with $124 \mathrm{k}$ polygons (point-surface collision detection) on a standard low-end PC $(400 \mathrm{MHz}$ Pentium II). Note that these figures are for detection of first contact; as long as contact is maintained collision detection tests times are fairly constant with the number of polygons afterwards.

\subsection{Simulation of a push-button}

The main interaction between a driver and a car, especially if we consider the dashboard, is done through the use of commands which basically consist in pressing or turning devices. At the very first level, we have decided to focus on a single push-button because it is already a challenge as we will see further and because it seemed rather simpler because of its small dimensions. Simulating a clutch pedal for instance would require bigger and stronger devices whereas we hoped to be able to use common FF hardware.

\subsubsection{Experiments}

Our scope is to verify that the haptic sense (force feedback in particular) felt by one's fingertip while pressing a real button, can be reproduced. Two different experiments were planned:

- For the first experiment, we have used the haptic device Phantom Desktop, for exploring and interacting with a VE in which a button is dynamically represented. There is a visual representation of the button and we can easily modify its course and stiffness. In that way we compare a nominal configuration with different ones, in order to define the JND.

- The second experiment is based on the use of an "adjustable" button, i.e. a button whose force is not created by real spring and damping elements, but is calculated by a computer and applied through a haptic interface.

\subsubsection{Experimental device}

Our experimental device consists of a box, that has attached on one of his sides 2 buttons. The first button is a real one, exactly as used on the dashboard of an automobile. The second is an "adjustable" one, whose force is controlled by the Phantom Desktop. The Phantom Desktop has 6 dof ( 3 of them active) and as a consequence is a redundant system in relation with the current application (we need only the 1 dof for the translation of the button). This choice was made, because it was the sole haptic interface in our possession and the software necessary for the control of such a device was already available with it (see photo in Fig. 6).

\subsubsection{Preliminary results}

It quickly appeared that the Phantom was not the adequate tool for two main reasons:

- The Phantom is a 6 dof system whereas one is enough (a linear engine for instance) and the other dofs provide us with some undesired vibrations.

- The Phantom is not able to reproduce the necessary forces and the feeling of the button is not realistic; the current trend in the car market is that the course of the buttons are getting shorter and the precision of the built system must be higher. Moreover, Phantom highest forces are obtained only on peaks whereas it would be necessary to have them continuously.

For these reasons, we have decided to build a dedicated system using a single degree-of-freedom which would sustain the necessary forces. The details of this system cannot be disclosed because it is adapted to the ranges of forces and torques that are actually used by the car manufacturer and therefore kept secret. The results of the experiments allow us to assume that we are able to simulate the behavior of a push-button.

\subsubsection{Next steps: putting the button in position and driving}

Putting the button in position without the dashboard simply requires specific hardware to maintain it into position and is quite an easy task. What is quite more challenging is to integrate this button into a virtual dashboard and lastly in a car simulator. At this stage we will have to address the display device issue: if you use an HMD, the user will not see the FF devices which will necessarily be close to him but will not see his arms and hands either which can be annoying for the experiment. If we use projective displays, the problem will be opposite, the user will be able to see his hands but will also see the FF device! We have to make further experiments to be able to take a decision.

In the mean time, we are developing a specific forcefeedback device dedicated to the ergonomic study of a car dashboard. Integrating it in a complete simulator adds up constraints because of the required physical integration of the components of the simulator. Moreover, numerous commands have to be either simulated or physically introduced into the simulator and all systems will have to interact together!

\subsection{Early conclusions on the use of FF devices for ergonomic studies}

It is of course too early to make a general conclusion on the use of force-feedback devices in the field of ergonomic studies but it seems to be quite promising as long as several problems will be overcome:

- The ideal generic force-feedback interface for ergonomic studies does not exist yet. Generic FF devices usually not only present the user low forces and 


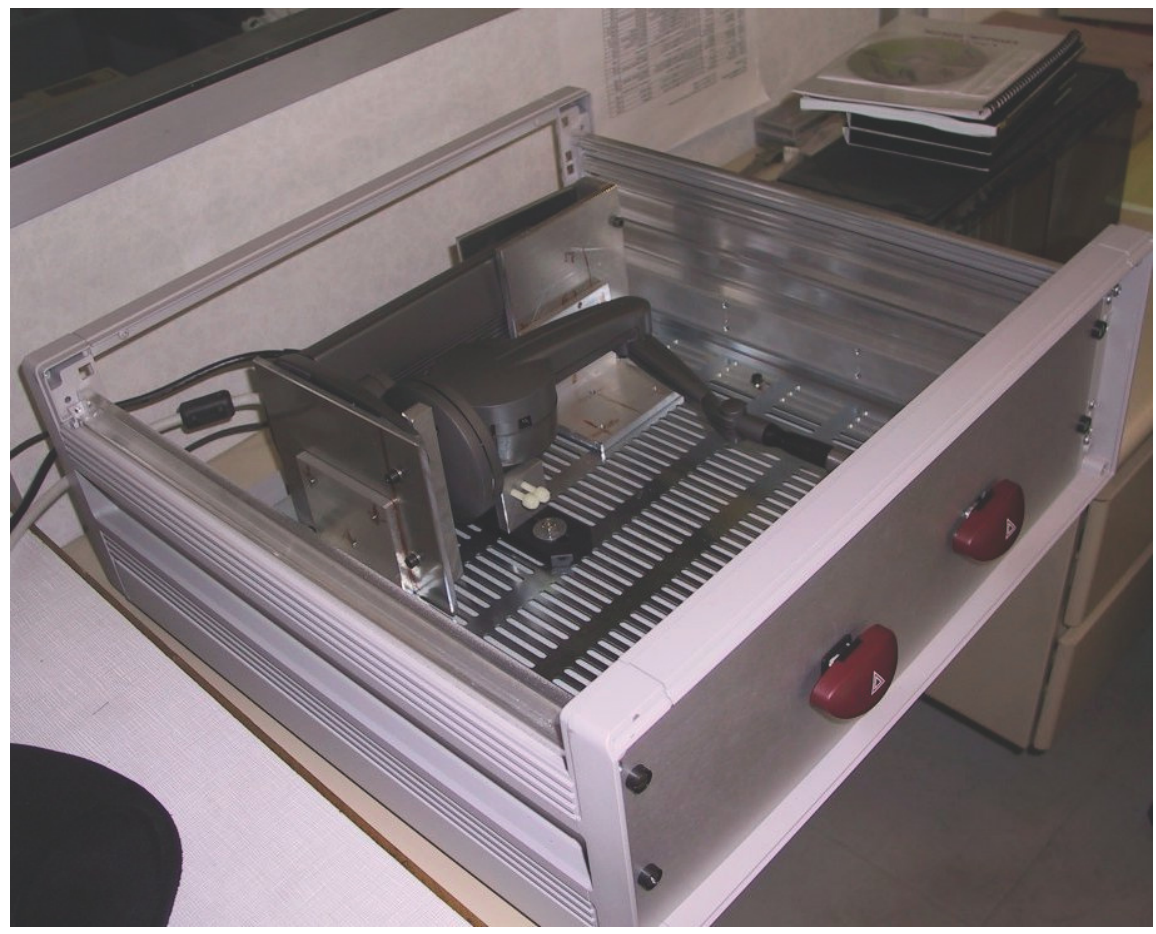

Fig. 6. Photo of the experimental device.

torques (only in peaks, not continuous most of the time) but also have too small workspaces.

- The next challenge is mainly on the software side: collision detection algorithms have to be much more efficient in order to be able to detect several contacts between the user and the virtual environment.

- The possible presence of FF devices into the visual field of the user raises new issues that must be studied further to determine which functions will be affected with respect to immersion of the user.

\section{Conclusion}

In this paper, we have presented the work undertaken at the Robotics Center in the field of VR for product design and manufacturing. After having presented our realization for design review and simple ergonomic studies, we stressed out that there were still some room for further research both in hardware and software.

There is also room for more research in the ergonomics field. Within the PERF-RV project we are working with a French car manufacturer and the CEA/LIST on the ergonomic study of a car dashboard. The main issues are the design of specific force-feedback devices for the present ones do not meet the requirements and many improvements in the collision detection algorithms that must be able to sustain $\mathrm{kHz}$ rates with hundred of thousands polygons.

Acknowledgements. This work was partially supported by Sommer Allibert Automotive Industry and the RNTL/PERFRV project.

\section{References}

[1] G. Moreau, P. Fuchs, Virtual Reality in the design process: application to design review and ergonomic studies, Proceedings of European Simulation Symposium, Marseille, France, 2001, pp. 123-130

[2] G. Moreau, P. Fuchs, Stereoscopic displays for Virtual Reality in the car manufacturing industry: application to design review and ergonomic studies. Proceedings of SPIE, Stereoscopic Displays \& Virtual Reality Systems IX, Vol. 4660B, San José, CA, 2002

[3] D. Paillot, F. Merienne, M. Neveu, J.P. Frachet, Virtual immersive review for car design, Proceedings of SPIE, Stereoscopic Displays \& Virtual Reality Systems X, Vol. 5006B, Santa Clara, CA, 2003

[4] PERF-RV, bureau d'études du futur, RNTL Framework, http://www . perfrv.org

[5] P. Biermann, B. Jung, M. Latoschik, I. Wachsmut, Virtuelle Werkstatt: a platform for multimodal assembly in VR, Proceedings of Virtual Reality International Conference 2002, Laval, France, 2002

[6] R. Steffan, U. Schull, T. Kuhlen, Integration of virtual reality based assembly simulation into CAD/CAM environments, IEEE, Aachen, Germany, 1998, pp. 2535-2537

[7] P. Fuchs, G. Moreau, B. Arnaldi, J.M. Burkhardt, A. Chauffaut, S. Coquillant, S. Donikian, T. Duval, J. Grosjean, F. Harrouet, E. Klinger, D. Lourdeaux, D. Mellet d'Huart, A. Parjic, I.P. Papin, P. Stergiopoulos, J. Tisseau, I. Viaud-Delmon, Le traité de la Réalité Virtuelle, Les Presses de l'École des mines de Paris, deuxième édition, 2 volumes, 2003 http://www-caor.ensmp.fr/interlivre

[8] C. Wheatstone, On some remarkable, and hitherto unobserved, phenomena of binocular vision. Philosophical transactions of the Royal Society of London, 371(94) 
[9] N. Valyus, Stereoscopy, Focal Press, 1962

[10] V. Moulet, Fonctionnalités attendues pour un logiciel d'ergonomie pour la conception de l'habitacle d'un véhicule, Journée spécialisée, modèles numériques pour la conception de produits, INRETS Lyon-Bron, 2000

[11] C. Tzafestas, Synthèse de retour kinesthésique et perception haptique lors de taches de manipulation. Ph.D. thesis, University of Paris 6, 1998

[12] L.A. Jones, I.W. Hunter, Human Operator Perception of Mechanical Variables and their Effects on Tracking Performance, Proceedings of the 1st Symposium on Haptic Interfaces for Virtual Environment and Teleoperator Systems, ASME Dynamic Systems and Control Division, Vol. 42, Anaheim, CA, USA, 1992, pp. $49-53$

[13] H.Z. Tan, X.D. Pang, N.I. Durlach, Manual Resolution of Length, Force, and Compliance, Proceedings of the 1st Symposium on Haptic Interfaces for Virtual Environment and Teleoperator Systems, ASME Dynamic Systems and Control Division, Vol. 42, Anaheim, CA, USA, 1992, pp. $13-18$

[14] T. Brooks, Telerobotic response requirements, IEEE International Conference on systems, man and cybernetics, Los Angeles, CA, USA, 1990, pp. 113-120

[15] P. Stergiopoulos, G. Moreau, D. Lourdeaux, P. Fuchs, Collision detection for haptic interactions with solid models and prototypes, Virtual Reality International Conference, Laval, France, 2002, pp. 151-158

[16] M. Held, ERIT: a collection of efficient and reliable intersections tests, ACM Journal of Graphics Tools (1997), (2) 4:25-44

[17] P. Stergiopoulos, G. Moreau, M. Ammi, P. Fuchs, A framework for the haptic rendering of the human hand. IEEE Virtual Reality Haptics Symposium, Los Angeles, CA, USA, 2003, pp. 340-347 